\title{
Star Position Estimation Improvements for Accurate Star Tracker Attitude Estimation
}

\author{
Tjorven Delabie* \\ K.U. Leuven, Heverlee, Vlaams Brabant, 3001, Belgium
}

\begin{abstract}
This paper presents several methods to improve the estimation of the star positions in a star tracker, using a Kalman Filter. The accuracy with which the star positions can be estimated greatly influences the accuracy of the star tracker attitude estimate. In this paper, a Kalman Filter with low computational complexity, that can be used to estimate the star positions based on star tracker centroiding data and gyroscope data is discussed. The performance of this Kalman Filter can be increased by adjusting its parameters using certain available information. Using information such as the power in the star signal or the shape of the signal, the noise values in the filter can be adjusted to improve the star position estimate. Furthermore, the filter also estimates the uncertainty on the star positions. These uncertainties can be used to assign more importance to stars with lower position uncertainty in the cost function of the attitude estimation algorithm. The Kalman Filter with these improvements was implemented and tested with simulated star data. Results show that the attitude estimation error is reduced significantly. This results in a more accurate attitude determination and control system which allows to perform more demanding missions.
\end{abstract}

\section{Nomenclature}

$(\hat{)} \quad$ Estimated value.

$\left(\omega_{x}, \omega_{y}, \omega_{z}\right)$ Rotational velocities of the spacecraft.

(.) Propagated value.

$(i, j, k)$ Unit vector coordinates of the star.

$\left(w_{i_{x}}, w_{i_{y}}\right) \quad$ Separate weights for the $x$ - and $y$-coordinates.

$(x, y)$ Coordinate of a star in the image frame.

$\left(x_{b}, y_{b}\right)$ Star centroid coordinates.

$\left(z_{x_{k}}, z_{y_{k}}\right)$ Measured state.

$\beta \quad$ Gyroscope bias.

$\Delta T \quad$ Time between two measurement steps.

$\eta_{1} \quad$ Angular Random Walk noise.

$\eta_{2} \quad$ Rate Random Walk noise.

$\Gamma \quad$ Cost value.

$\mathbf{b}_{i} \quad$ A unit vector of star $i$, observed in the spacecraft body frame.

$\mathbf{H}_{\mathbf{k}} \quad$ Observation matrix.

$\mathbf{K}_{\mathbf{k}}$ Kalman Gain.

$\mathbf{P}_{\mathbf{k}} \quad$ Covariance matrix.

$\mathbf{q}(\mathbf{0})$ Initial attitude quaterion.

$\mathbf{Q}_{\mathbf{k}} \quad$ Process noise covariance matrix.

q Attitude quaterion.

$\mathbf{R}_{\mathbf{k}} \quad$ Measurement noise covariance matrix.

$\mathbf{r}_{i} \quad$ A unit vector of star $i$, observed in a reference frame.

$\phi \quad$ Roll angle.

$\sigma_{r o} \quad$ Read noise standard deviation.

*PhD Researcher, Department of Mechanical Engineering, Celestijnenlaan 300B, Member AIAA 
$\sigma_{s n, i, j} \quad$ Shot noise standard deviation.

$\sigma_{\text {tot }} \quad$ Total centroiding error standard deviation.

$\theta_{e} \quad$ The angle in arc seconds between the true quaternion - $q_{t}$ - and the estimated quaterion - $q_{e}$.

$D C \quad$ Dark Current Signal.

$f \quad$ Focal length.

$N \quad$ Peak number of electrons.

$N_{\text {bits }} \quad$ Number of bits in the camera read-out electronics.

$n_{\text {pixels }}$ Number of pixels in one row or column of the camera.

$n_{\text {stars }}$ Number of stars in the image.

pow Power in the star signal.

$q_{e} \quad$ The quaternion representing the attitude estimated by the attitude estimation algorithm.

$q_{t} \quad$ The quaternion representing the true attitude.

$t_{x} \quad$ Distance over which the database stars are translated in the $x$-direction.

$t_{y} \quad$ Distance over which the database stars are translated in the $y$-direction.

$V_{x, y} \quad$ Measured pixel value at coordinates $(x, y)$.

$w_{i} \quad$ Non-negative weight of star $i$.

$\left(\sigma \operatorname{ramp}_{x}, \sigma \operatorname{ramp}_{y}\right)$ Rate of increase of the star signal standard deviations per distance from the center. $\left(\sigma_{x}, \sigma_{y}\right)$ Star signal standard deviation.

$\left(\sigma_{\text {base }_{x}}, \sigma_{\text {base }_{y}}\right)$ Base value for the star signal standard deviations.

\section{Introduction}

In many spacecraft missions, accurate attitude determination is a crucial aspect. Of the various sensors to determine the attitude of a spacecraft, the star tracker is the most accurate. ${ }^{1}$ This sensor estimates the attitude of the spacecraft by comparing star positions in an image taken on board of the spacecraft with a database of known star positions. The attitude estimation error of a star tracker depends for a large part on the accuracy of the position estimates of the stars on the image plane. ${ }^{2}$

The star positions are measured by the centroiding algorithm. In order to facilitate the determination of the centroids with subpixel accuracy, the optics of the star tracker are slightly defocused so that the star light is spread out over several pixels. ${ }^{3}$ A range of different centroiding algorithms has been developed. An overview of the most common algorithms is given in ${ }^{4}$ and. ${ }^{5}$ The most accurate centroiding algorithms rely on fitting a point spread function (PSF) to the measured pixel data. ${ }^{6}$ The fastest centroiding algorithms generally calculate a center of gravity to determine the centroid of each star. ${ }^{5}$

The accuracy of the star position estimates can be increased by using a state estimator such as a Kalman Filter. This was already demonstrated in, ${ }^{7}$ where a Kalman Filter is developed for this problem. This filter propagates the star positions using gyroscope measurements. In this paper we will first present another method to estimate the star positions with a Kalman Filter. The equations of this estimator are more simple and the state estimator is therefore less computationally complex.

In this paper, we show that there are additional benefits to incorporating a Kalman Filter in the centroiding procedure. Some sources of information that are available in the centroiding procedure (star signal power, width of the point spread function,...) can give an indication of the centroiding error. Stars with lower power will have a lower signal-to-noise ratio and can therefore be expected to have a larger centroiding error. This information can be used to adapt the noise values in the Kalman Filter, leading to a more accurate Kalman gain and hence more accurate star position estimate.

Furthermore, the Kalman Filter not only estimates the star positions, it also estimates the uncertainty on these star positions. In this way, the Kalman Filter provides a convenient method to determine the weights used in the attitude estimation cost function. The attitude estimations generally incorporate weights assigned to each star pair. ${ }^{8}$ This way, a star which has higher uncertainty on its position can be assigned a higher weight. The recently developed AIM algorithm ${ }^{9}$ allows to easily assign different weights to the $x$ - and $y$ coordinate of each star, leading to an even more accurate weighting.

Adjusting the noise values in the Kalman Filter and using the uncertainty estimates to determine weights, can increase the accuracy of the star tracker attitude estimate. This leads to a more accurate and performant attitude determination and control system. In section II, the basic Kalman Filter procedure to estimate star positions is presented. After this, section III discusses several sources of available information that allow to improve our knowledge of the expected centroiding error. Section IV discusses the use of the Kalman Filter 
to provide weights for the attitude estimation algorithm. In section $\mathrm{V}$, the simulations results of the different cases are discussed. Finally, section VI gives a conclusion.

\section{Centroiding Kalman Filter}

Typically, the star tracker functions as a 'black box' in the spacecraft. It takes an image of the star field as input and outputs the estimated attitude quaternion. All the algorithms in the star tracker are executed without inputs from the other sensors. The output of the star tracker is then often combined with the information of the on-board gyroscopes in e.g. a Kalman Filter. The purpose of fusing different sensor outputs is to reduce the uncertainty of the estimated attitude and to improve the accuracy.

In this section, we will incorporate the gyroscope data in the star tracker attitude estimation procedure. Specifically, the gyroscope sensor data will be used in the Centroiding step. The rate information will allow us to estimate the current position of each of the stars in the image plane based on the previous position estimates and the angular rate of the spacecraft. The star positions obtained by doing this, referred to as the propagated star positions will be used together with the star positions measured by the star tracker itself, to obtain more accurate star positions and hence a more accurate attitude estimate. A Kalman Filter will be used to fuse the propagated and measured star positions. First, the propagation step of the Kalman Filter will be discussed.

\section{A. Propagation}

The star positions on the image plane change due to the rotation of the spacecraft in space. Based on the rotational velocities of the spacecraft, the sampling time, and the estimated position of the stars in the previous time step, the star positions in the current time step can be calculated. Because the rotational velocities of a spacecraft are typically low (in the order of tenths to hundredths of a degree per second) and because the sampling frequency of a star tracker is typically around 1 to $10 \mathrm{~Hz}$, the angle over which the spacecraft has rotated in between star tracker measurements is generally small. Therefore, the propagation step in the presented Kalman Filter uses a rotation matrix based on a small-angle approximation. Other methods to perform the propagation exist, ${ }^{7}$ but the required computations are significantly more computationally expensive.

To obtain the propagation equations, the focal plane coordinates of the star of the previous time step $(k-1)$, are first transformed to unit vectors using a simple pinhole model: ${ }^{10}$

$$
\left[\begin{array}{l}
i_{k-1} \\
j_{k-1} \\
k_{k-1}
\end{array}\right]=\left[\begin{array}{l}
\frac{\frac{-x_{k-1}}{f}}{\sqrt{1+\left(\frac{x_{k-1}}{F}\right)^{2}+\left(\frac{y_{k-1}}{f}\right)^{2}}} \\
\frac{\frac{y_{k-1}}{f}}{\sqrt{1+\left(\frac{x_{k-1}}{F}\right)^{2}+\left(\frac{y_{k-1}}{f}\right)^{2}}} \\
\frac{1}{\sqrt{1+\left(\frac{x_{k-1}}{f}\right)^{2}+\left(\frac{y_{k-1}}{f}\right)^{2}}}
\end{array}\right] .
$$

With $(x, y)$, the coordinate of the star in the image frame, $f$ the focal length and $(i, j, k)$, the unit vector coordinates of the star. In the further derivation, the term in the denominator will be of little value and to improve readability, we will replace it with a short-hand notation $c$ :

$$
\left[\begin{array}{c}
i_{k-1} \\
j_{k-1} \\
k_{k-1}
\end{array}\right]=\left[\begin{array}{c}
\frac{-x_{k-1}}{f \cdot c} \\
\frac{y_{k-1}}{f_{1} c} \\
\frac{1}{c}
\end{array}\right] .
$$

In between star tracker measurements, the spacecraft rotates with a rotational velocity that is measured by the gyroscopes. The star vectors will also rotate with this rotational velocity. In order to obtain the rotated star vectors, the vectors of the previous time step are multiplied by a rotation matrix containing the rotational velocities, multiplied with the sampling time. Because the angles are small as was mentioned 
above, a rotation matrix with a small-angle approximation was used. the rotated vectors are then calculated as:

$$
\left[\begin{array}{l}
i_{k} \\
j_{k} \\
k_{k}
\end{array}\right]=\left[\begin{array}{ccc}
1 & \omega_{z} \Delta T & -\omega_{y} \Delta T \\
-\omega_{z} \Delta T & 1 & \omega_{x} \Delta T \\
\omega_{y} \Delta T & -\omega_{x} \Delta T & 1
\end{array}\right]\left[\begin{array}{c}
\frac{-x_{k-1}}{f \cdot c} \\
\frac{y_{k-1}}{f \cdot c} \\
\frac{1}{c}
\end{array}\right]
$$

where $\Delta T$ is the time step in between two star tracker measurements. To obtain the focal plane coordinates from these unit vector coordinates, a pinhole model is used:

$$
\left[\begin{array}{l}
x_{k} \\
y_{k}
\end{array}\right]=f .\left[\begin{array}{c}
-\frac{i_{k}}{k_{k}} \\
\frac{j_{r}}{k_{r}}
\end{array}\right]=f \cdot\left[\begin{array}{c}
\frac{x_{k-1}-\omega_{z} \Delta T y_{k-1}+f \omega_{y} \Delta T}{f-\omega_{y} \Delta T x_{k-1}-\omega_{x} \Delta T y} \\
\frac{\omega_{z} \Delta T x_{k-1}+y_{k-1}+f \omega_{x} \Delta T}{f-\omega_{y} \Delta T x_{k-1}-\omega_{x} \Delta T y}
\end{array}\right] .
$$

Working towards a notation that is convenient for use in a Kalman Filter, we obtain the following expressions to calculate the star positions at the current time step, based on the estimate of the previous time step and the measured angular velocity:

$$
\left[\begin{array}{c}
\tilde{x}_{k} \\
\tilde{y}_{k}
\end{array}\right]=\frac{f}{f-\omega_{y} \Delta T \hat{x}_{k-1}-\omega_{x} \Delta T \hat{y}_{k-1}} \cdot\left[\begin{array}{cc}
1 & -\omega_{z} \Delta T \\
\omega_{z} \Delta T & 1
\end{array}\right]\left[\begin{array}{c}
\hat{x}_{k-1} \\
\hat{y}_{k-1}
\end{array}\right]+\frac{f^{2}}{f-\omega_{y} \Delta T \hat{x}_{k-1}-\omega_{x} \Delta T \hat{y}_{k-1}}\left[\begin{array}{c}
\omega_{y} \Delta T \\
\omega_{x} \Delta T
\end{array}\right]
$$

In this notation, a hat $(\hat{.})$ on a symbol denotes an estimated value, while a tilde ( $($.$) denotes a propagated$ value.

Because the rotational velocities, time step between measurements and $x$ - and $y$ coordinates are typically low, the second and third term in the denominators given above are often negligible compared to $f$. Equation 5 can then be simplified to:

$$
\left[\begin{array}{c}
\tilde{x}_{k} \\
\tilde{y}_{k}
\end{array}\right]=\left[\begin{array}{cc}
1 & -\omega_{z} \Delta T \\
\omega_{z} \Delta T & 1
\end{array}\right]\left[\begin{array}{c}
\hat{x}_{k-1} \\
\hat{y}_{k-1}
\end{array}\right]+f\left[\begin{array}{c}
\omega_{y} \Delta T \\
\omega_{x} \Delta T
\end{array}\right]
$$

Using the simple equation 6 , the positions of the stars in the focal plane can be propagated with a very low computational cost.

\section{B. Measurement}

The star tracker measures the position of each star based on the measured pixel values with a centroiding algorithm. To facilitate the determination of the centroids with subpixel accuracy, the optics of the star tracker are slightly defocused so that the star light is spread out over several pixels. ${ }^{3}$

A variety of different algorithms to determine star centroids has been developed. An overview of the most common of these algorithms is given in $^{4}$ and. ${ }^{5}$ The most accurate of these centroiding algorithms rely on fitting a point spread function (PSF) to the measured pixel data. ${ }^{6}$ This point spread function is the impulse response of the imaging system ${ }^{11}$ and can be very accurately modeled by a Gaussian profile. ${ }^{12}$ While these algorithms determine the star centroid with great accuracy, they are computationally expensive because they use an iterative least squares function fitting approach. ${ }^{4}$ Another approach is to calculate the center of gravity or variations on this center of gravity ${ }^{5}$ to determine the star centroids. Algorithms using this approach are considerably less computationally expensive, but calculate the centroids with lower accuracy. ${ }^{4}$

The star tracker directly measures the $x$ - and $y$-position of the star in the image field. The measured star positions at time step $k$ are referred to as:

$$
\left[\begin{array}{c}
z_{x_{k}} \\
z_{y_{k}}
\end{array}\right]
$$




\section{Covariance Computation}

Together with the state estimate, the Kalman Filter also estimates the covariance. First, the variance is propagated:

$$
\begin{aligned}
\tilde{\mathbf{P}}_{\mathbf{k}} & =\left[\begin{array}{ll}
\tilde{P}_{x x_{k}} & \tilde{P}_{x y_{k}} \\
\tilde{P}_{y x_{k}} & \tilde{P}_{y y_{k}}
\end{array}\right]=\left[\begin{array}{cc}
1 & -\omega_{z} \Delta T \\
\omega_{z} \Delta T & 1
\end{array}\right]\left[\begin{array}{ll}
\hat{P}_{x x_{k-1}} & \hat{P}_{x y_{k-1}} \\
\hat{P}_{y x_{k-1}} & \hat{P}_{y y_{k-1}}
\end{array}\right]\left[\begin{array}{cc}
1 & -\omega_{z} \Delta T \\
\omega_{z} \Delta T & 1
\end{array}\right]^{T} \\
& +\left[\begin{array}{ccc}
0 & f \Delta T & -y \Delta T \\
f \Delta T & 0 & x \Delta T
\end{array}\right]\left[\begin{array}{ccc}
\sigma_{\omega x_{k}}{ }^{2} & 0 & 0 \\
0 & \sigma_{\omega y_{k}}{ }^{2} & 0 \\
0 & 0 & \sigma_{\omega z_{k}}{ }^{2}
\end{array}\right]\left[\begin{array}{ccc}
0 & f \Delta T & -y \Delta T \\
f \Delta T & 0 & x \Delta T
\end{array}\right]^{T} .
\end{aligned}
$$

Here, $\sigma_{\omega x_{k}}, \sigma_{\omega y_{k}}$, and $\sigma_{\omega z_{k}}$ are the standard deviations of the gyroscope noise around the three axes and form the matrix $\mathbf{Q}_{\mathbf{k}}$.

The Kalman gain $K_{k}$ is calculated as:

$$
\mathbf{K}_{\mathbf{k}}=\tilde{\mathbf{P}}_{\mathbf{k}} \mathbf{H}^{T}\left[\mathbf{H} \tilde{\mathbf{P}}_{\mathbf{k}} \mathbf{H}^{T}+\left[\begin{array}{cc}
\sigma_{\text {stx }}^{2} & 0 \\
0 & \sigma_{\text {styk }}^{2}
\end{array}\right]\right]^{-1},
$$

where $\sigma_{s t x_{k}}$ and $\sigma_{s t y_{k}}$ are the standard deviations of the star tracker noise in the $x$ - and $y$-direction and form the matrix $\mathbf{R}_{\mathbf{k}}$. The matrix $\mathbf{H}$ is a unit matrix. The covariance matrix is then updated as:

$$
\hat{\mathbf{P}}_{\mathbf{k}}=\left(\mathbf{I}-\mathbf{K}_{\mathbf{k}} \mathbf{H}\right) \tilde{\mathbf{P}}_{\mathbf{k}}\left(\mathbf{I}-\mathbf{K}_{\mathbf{k}} \mathbf{H}\right)^{T}+\mathbf{K}_{\mathbf{k}} \mathbf{R}_{\mathbf{k}} \mathbf{K}_{\mathbf{k}}{ }^{T} .
$$

\section{State update}

The state can then be updated. Using the Kalman gain $K_{k}$, which is calculated using the estimated noise values of both gyroscope and centroiding measurement, the state can be updated. The estimated star positions are calculated using equation 11 :

$$
\left[\begin{array}{c}
\hat{x}_{k} \\
\hat{y}_{k}
\end{array}\right]=\left[\begin{array}{c}
\tilde{x}_{k} \\
\tilde{y}_{k}
\end{array}\right]+\mathbf{K}_{\mathbf{k}}\left(\left[\begin{array}{c}
z_{x_{k}} \\
z_{y_{k}}
\end{array}\right]-\mathbf{H}\left[\begin{array}{c}
\tilde{x}_{k} \\
\tilde{y}_{k}
\end{array}\right]\right) .
$$

These estimated centroids can then be matched with their corresponding database stars and an attitude quaternion can be calculated. In the following sections, we will discuss adaptations on this approach which can improve the performance of the attitude estimation procedure.

\section{Adjusting the Filter to Noise}

Typically in Kalman Filtering, the values of the process noise, $\mathbf{Q}_{\mathbf{k}}$ and the measurement noise $\mathbf{R}_{\mathbf{k}}$ are set at the initialization and are not changed during the filtering sequence. Therefore, values are chosen for the noise standard deviations which are representative for the range of cases that the filter will encounter.

However, it is possible to adapt the noise matrices during operation of the filter. In the procedure that will be discussed next, the measurement noise values will be adjusted for each star, based on certain information of those measurements. This additional information allows to improve our knowledge of the values of the matrix $\mathbf{R}_{\mathbf{k}}$ for each star separately. This in turn allows to calculate a more accurate Kalman gain $\mathbf{K}_{\mathbf{k}}$, which then results in an improved fusing of the sensor data and therefore in a higher accuracy of the star position estimation. In the rest of this section, we will discuss sources of information that could be used to improve the knowledge of the measurement noise values, and how they can be used. In section $\mathrm{V}$, these different sources will be analysed and their value to improve the knowledge of the measurement noise values will be assessed. 


\section{A. The Power of the Signal}

Stars have varying magnitudes and the power of the signal of the star, detected by the star tracker camera, will therefore vary. Stars with a higher magnitude are less bright and will therefore result in a signal with lower power.

On top of the star signal, a star tracker camera is subjected to various noise sources. ${ }^{13}$ These noise sources can be dependent on the star signal, (e.g. Shot noise) or independent, such as Read noise or Dark Current. The presence of this noise has an adverse effect on the centroiding accuracy of the star tracker. The higher the signal-to-noise ratio, the higher the centroiding accuracy will be. We can therefore expect that the centroiding algorithm will estimate the positions of stars with a higher signal-to-noise ratio with a lower error.

The power in a star signal can be calculated approximately by summing the pixel values of the window that was chosen around the star. This information is readily available and the computational cost to determine this value is low. In general, stars with higher power will have a larger signal-to-noise ratio and their positions will be estimated with higher accuracy. In section $V$, the effect of the signal power on the centroiding accuracy will be analyzed in detail.

\section{B. The Point Spread Function Width}

To improve the accuracy of the centroiding algorithm, the star light is spread out over several pixels. The resulting star signal can be accurately modeled by a Gaussian function. An important parameter of this function is the width of this Gaussian (see Figure 1). This width is often described by the Full Width Half Maximum (FWHM) or by the standard deviation of the gaussian function.

Due to the optics, this width varies over the image plane. Generally, the Point Spread Function is wider towards the edge of the image. The centroiding accuracy depends on this width and decreases with increasing width. When a centroiding algorithm is used that fits a Gaussian profile through the data, the PSF width is also estimated. This width can vary for the $x$ - and $y$-direction, depending on the position of the star on the image plane. The most accurate centroiding algorithms fit a Gaussian function through the pixel data ${ }^{13}$ and estimate the PSF widths in any case, so no extra computations are required to obtain these values. In section $\mathrm{V}$, the effect of the PSF width on the centroiding accuracy will be examined in detail.

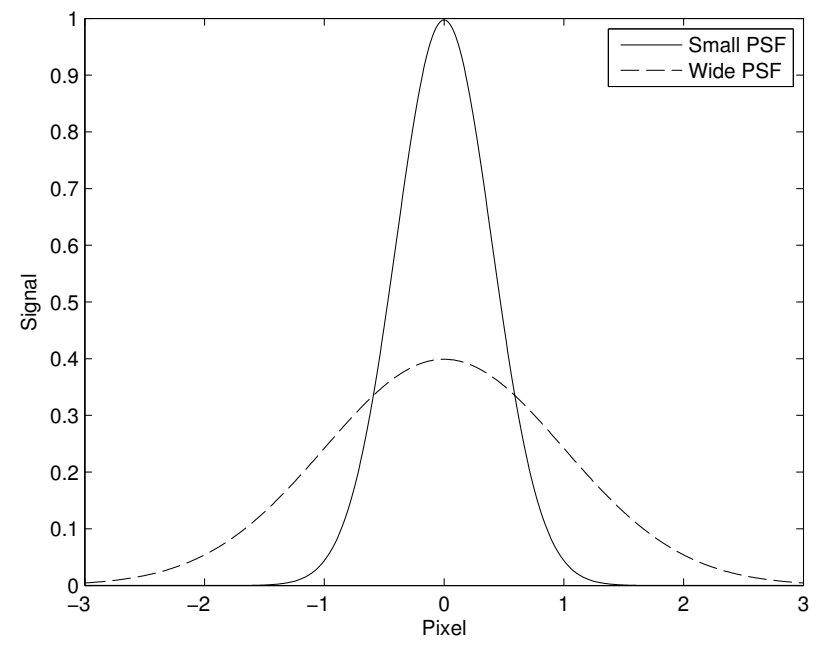

Figure 1: Two Gaussian Point Spread Functions with different widths.

Other sources of information could be used to improve our knowledge of the measurement noise. When the centroid is estimated by fitting a model through the pixel values, the residual of the cost function could be a measure for the measurement noise. 
The improved knowledge of the measurement noise leads to a more accurate Kalman gain and therefore an improved fusing of the sensor data. On top of this beneficial effect, the improved knowledge also increases the accuracy of the covariance estimate. Because the measurement noise is known with higher accuracy, the uncertainty on the star position estimate is estimated with higher accuracy. In the next section, it is shown that the knowledge of the uncertainty on the star position can improve the accuracy of the star tracker.

\section{Uncertainties and Weights}

On top of providing star position estimates with increased accuracy, the Kalman Filter yields an additional advantage. Next to estimating the state, the Kalman Filter also estimates the uncertainty on the state estimates. The knowledge of the star position uncertainty can be of value in the attitude estimation procedure. After the star positions have been estimated, they are paired with their corresponding database stars. The combinations of measured stars and database stars form the input for the tracking algorithm. Almost all state of the art tracking algorithms estimate the spacecraft attitude by solving Wahba's problem:

$$
L(A)=\frac{1}{2} \sum_{i=1}^{n} w_{i}\left|\mathbf{b}_{i}-A \mathbf{r}_{i}\right|^{2},
$$

where $\mathbf{b}_{i}$ are the unit vectors observed in the spacecraft body frame, $\mathbf{r}_{i}$ are the corresponding unit vectors in a reference frame and $w_{i}$ are non-negative weights.

A recent algorithm, referred to as $\mathrm{AIM},{ }^{9}$ solves the $2 \mathrm{D}$ equivalent of this problem to determine the attitude:

$$
\Gamma\left(\phi, t_{x}, t_{y}\right)=\sum_{i=1}^{n_{s}} w_{i}\left(\left(x_{i}-\bar{x}_{i} \cos (\phi)+\bar{y}_{i} \sin (\phi)-t_{x}\right)^{2}+\left(y_{i}-\bar{x}_{i} \sin (\phi)-\bar{y}_{i} \cos (\phi)-t_{y}\right)^{2}\right) .
$$

In this function, $w_{i}$ is the weight given to star $i,\left(x_{i}, y_{i}\right)$ are the coordinates of observed star $i$ in the focal plane, $\left(\bar{x}_{i}, \bar{y}_{i}\right)$ are the coordinates of the corresponding database star $i$ in the focal plane, $\phi$ is the angle over which the database stars are rotated with respect to the origin of the frame in which the database coordinates are described, $t_{x}$ and $t_{y}$ are the distances over which the database stars are translated in $x$ - and $y$-direction respectively, and $n_{s}$ is the number of stars used in the attitude estimation algorithm.

Both Wahba's problem and the AIM cost function assign weights to the distances between each measured star and corresponding database star. Based on these weights, the distances can be given more or less importance in the cost function. When the uncertainty on the position estimate of a measured star is lower, the star can be given higher importance and vice versa. This way, the attitude estimate is obtained with higher accuracy.

The uncertainties on the $x$ - and $y$-position of a measured star can be different, and both are estimated by the Kalman Filter. Because the AIM cost function is described in image plane coordinates, it allows to intuitively assign different weights to the $x$ - and $y$-coordinates of the star. The AIM algorithm therefore allows to take into account different uncertainty estimates on the $x$ - and $y$-coordinates. This allows to weigh the different distances with higher accuracy. The cost function to be solved for AIM is then:

$$
\Gamma\left(\phi, t_{x}, t_{y}\right)=\sum_{i=1}^{n_{s}}\left(w_{i_{x}}\left(x_{i}-\bar{x}_{i} \cos (\phi)+\bar{y}_{i} \sin (\phi)-t_{x}\right)^{2}+w_{i_{y}}\left(y_{i}-\bar{x}_{i} \sin (\phi)-\bar{y}_{i} \cos (\phi)-t_{y}\right)^{2}\right) .
$$

This new cost function leads to a slightly different solution for the AIM algorithm. Since the AIM algorithm is not the focus of this paper, only the changes as opposed to the original AIM algorithm ${ }^{9}$ are shown here. The variable $\phi$ is still calculated using the original cost function:

$$
\begin{aligned}
& t_{x}=s x x \cdot \cos (\phi)+s y x \cdot \sin (\phi)-s \bar{x} \\
& t_{y}=s x y \cdot \sin (\phi)+s y y \cdot \cos (\phi)-s \bar{y}
\end{aligned}
$$




$$
\begin{array}{rlrl}
s x x & =\sum_{n=1}^{n_{s}} w_{i_{x}} \cdot x_{i} & s y y & =\sum_{n=1}^{n_{s}} w_{i_{y}} \cdot y_{i} \\
s x y & =\sum_{n=1}^{n_{s}} w_{i_{y}} \cdot x_{i} & s \hat{x} & =\sum_{n=1}^{n_{s}} w_{i_{x}} \cdot \bar{x}_{i} \\
s y x & =\sum_{n=1}^{n_{s}} w_{i_{x}} \cdot y_{i} & s \hat{y}=\sum_{n=1}^{n_{s}} w_{i_{y}} \cdot \bar{y}_{i}
\end{array}
$$

The rest of the AIM algorithm derivation can be found in reference. ${ }^{9}$ The important part for this paper is that the AIM algorithm allows to take into account the uncertainties on $x$ - and $y$-coordinates of the stars separately.

\section{Simulations}

In this section, the simulation setup will be discussed first. After this, an analysis will be performed to examine how certain sources of information can allow to adjust the measurement noise in the Kalman Filter. After this, there is a brief discussion on adapting the weights in the tracking algorithm based on the uncertainty estimates of the star positions. Finally, simulations are performed to assess the attitude estimation accuracy using the Centroiding Kalman Filter with the various potential improvements mentioned above.

\section{A. Data Generation}

\section{Trajectory Generation}

The different implementations of the Centroiding Kalman Filter are tested with sequences of simulated gyroscope data and star image data.

First, an initial attitude $\mathbf{q}(\mathbf{0})$ and measurement sample time $\Delta T$ are defined. Then, rotational velocities $\omega_{x}, \omega_{y}$, and $\omega_{z}$, are simulated for all time steps. Based on the choice of these, one can simulate pointing maneuvers, slews, ...

The quaternion at each time step can then be calculated using differential equation: ${ }^{14}$

$$
\frac{d}{d t} \mathbf{q}=\frac{1}{2}[\Omega] \mathbf{q},
$$

With $\Omega$ as:

$$
\Omega=\left[\begin{array}{cccc}
0 & \omega_{z} & -\omega_{y} & \omega_{x} \\
-\omega_{z} & 0 & \omega_{x} & \omega_{y} \\
\omega_{y} & -\omega_{x} & 0 & \omega_{z} \\
-\omega_{x} & -\omega_{y} & -\omega_{z} & 0
\end{array}\right]
$$

\section{Gyroscope Data Generation}

The gyroscopes measure the angular rate of the spacecraft. These measurements are used to propagate the star positions in the Centroiding Kalman Filter. To obtain gyroscope measurements with realistic noise values, the gyroscope model of equation 21 was used. ${ }^{15}$

$$
\begin{array}{r}
u=\omega+\beta+\eta_{1} \\
\dot{\beta}=\eta_{2}
\end{array}
$$


The gyroscope measurements are increased with the bias and an Angular Random Walk (ARW). The ARW is considered to be a zero mean white Gaussian noise process. The bias itself is modeled as a random walk process referred to as the Rate Random Walk (RRW). This RRW is also considered to be a zero mean white Gaussian noise process. ${ }^{16}$

\section{Star Tracker Image Generation}

At each time step, a star image is generated. Stars are selected from the Hipparcos ${ }^{17}$ catalog and are modeled as elliptical Gaussian signals with power depending on the magnitude of the star: ${ }^{13}$

$$
V_{x, y}=N \int_{x-0.5}^{x+0.5} \frac{1}{\sqrt{2 \pi} \sigma_{x}} e^{-\frac{\left(x-x_{b}\right)^{2}}{2 \sigma_{x}^{2}}} \int_{y-0.5}^{y+0.5} \frac{1}{\sqrt{2 \pi} \sigma_{y}} e^{-\frac{\left(y-y_{b}\right)^{2}}{2 \sigma_{y}^{2}}}
$$

where $V_{x, y}$ is the measured pixel value at coordinates $(x, y), N$ is the peak number of electrons, the centroid of the star is at coordinates $\left(x_{b}, y_{b}\right)$, and $\sigma_{x}$ and $\sigma_{y}$ are the $x$ and $y$ standard deviations. These standard deviations generally increase with increasing distance from the center of the image as was explained in section III. A base-value for these values was chosen, $\sigma_{b_{a s e}}$ and $\sigma_{b_{a s e}}$, and these values were increase linearly with $x$ - and $y$-distance from the center of the image respectively.

The following noise sources are then added to the image:

- Dark Current: A constant signal $D C$

- Shot Noise: White noise with standard deviation equal to the square root of the signal $\left(\sigma_{s n, i, j}=\sqrt{V_{i, j}}\right)$

- Read Noise: White noise with standard deviation $\sigma_{r o}$

- Quantization Noise: The signal is rounded because of a limited number $\left(N_{\text {bits }}\right)$ of bits.

These noise sources were added as described in reference. ${ }^{13}$

\section{4. $\quad$ Used Values}

The values that were used to generate the maneuvers and data sets for the results given in the rest of the paper are given in table 1 . The simulated maneuver was a pointing maneuver.

\section{B. Processing}

The centroiding kalman filter and its adaptations were tested on the simulated data set. In each step, the generated data of that time step was called and processed.

The Kalman Filter is first initialized by choosing values for the $\mathbf{P}_{\mathbf{k}}$-matrix. These are set sufficiently high to indicate that there is a lot of uncertainty on the initial estimate. The Centroiding algorithm is called on the first image to initialize the star positions. The values in the noise matrices $\mathbf{Q}_{\mathbf{k}}$ and $\mathbf{R}_{\mathbf{k}}$ are determined by analysing the errors between the noise measurement data and the known true data.

From that point on, in each time step, the star positions of the previous time step are propagated using the generated noisy gyroscope values. After this, the centroiding algorithm measures the star centroids and the Kalman filter estimates the star position from these sources of information as discussed in section II. A fixed number of stars $n_{\text {stars }}$ is present in every image. When one of the stars leaves the image, a new star enters the image and can be tracked. When there is a change of stars, the $\mathbf{P}_{\mathbf{k}}$-matrix is reinitialized because there is again high uncertainty.

The attitude is calculated from the estimated stars position using the AIM algorithm. ${ }^{9}$ The attitude determination error was determined by calculating the angle in arc seconds between the estimated quaternion - $q_{e}$ - and the true quaternion - $q_{t}$ - as:

$$
\theta_{e}=2 a \cos \left(\mathbf{q}_{e} \cdot \mathbf{q}_{t}\right) / \pi * 180 * 3600
$$


Table 1: The values used to generate the data sets

\begin{tabular}{|c|c|c|}
\hline Name & Value & Unit \\
\hline Measurement frequency & 1 & $\mathrm{~Hz}$ \\
\hline Simulation time & 100 & $\mathrm{~s}$ \\
\hline$q_{0}$ & {$\left[\begin{array}{llll}1 & 0 & 0 & 0\end{array}\right]$} & - \\
\hline$\omega_{x}$ & white noise, $\sigma=0.005$ & $\mathrm{rad} / \mathrm{s}$ \\
\hline$\omega_{y}$ & white noise, $\sigma=0.005$ & $\mathrm{rad} / \mathrm{s}$ \\
\hline$\omega_{z}$ & white noise, $\sigma=0.005$ & $\mathrm{rad} / \mathrm{s}$ \\
\hline ARW & 0.05 & $\operatorname{arcsec} / \sqrt{s}$ \\
\hline RRW & 0.01 & $\operatorname{arcsec} / \sqrt{s^{3}}$ \\
\hline$n_{\text {pixels }}$ & 1024 & - \\
\hline$n_{\text {stars }}$ & 9 & - \\
\hline Field of view & $16.4 \times 16.4$ & ${ }^{\circ} x^{\circ}$ \\
\hline$\sigma_{\text {base }_{x}}$ & 1 & pixel \\
\hline$\sigma_{\text {base }_{y}}$ & 1 & pixel \\
\hline$\sigma \operatorname{ramp}_{x}$ & 0.1 & $1 / \mathrm{mm}$ \\
\hline$\sigma \operatorname{ramp}_{y}$ & 0.1 & $1 / \mathrm{mm}$ \\
\hline$D C$ & 100 & $e^{-}$ \\
\hline$\sigma_{r o}$ & 100 & $e^{-}$ \\
\hline$N_{\text {bits }}$ & $2^{10}$ & - \\
\hline
\end{tabular}

\section{Adjusting the Filter to Noise}

In this subsection, we first examine how available information in the star tracker can allow to improve the knowledge of the measurement noise. The two sources of information mentioned in section III will be examined. The relation between these sources of information and the centroiding error will be analyzed. After this, the generated data sets will be processed using both the normal Kalman Filter mentioned in section II, as the Kalman filter with the adaptations mentioned in section III. At this point, only the power in the signal was used to adjust the noise values.

\section{The Power of the Signal}

To simulate the relationship between the signal power of a star and the centroiding error, simulations were performed with a wide range of stars with different magnitudes. The centroiding algorithm was executed for these images and the $x$-coordinate centroiding error was set out in function of the star magnitude. The result for the $y$-coordinate was similar. The result is shown in figure 2 .

As can be seen from figure (a) of figure 2, the centroiding error decreases with increasing signal power. This is because the signal-to-noise ratio increases, allowing to more accurately determine the centroid. At very low signal powers, the centroiding error increases considerably. It is clear from this figure that the signal power of a star can provide useful information to estimate the centroiding error. In order to use this information in our Kalman Filter, a function was fitted through the standard deviation of the total centroiding error in function of the signal power (figure 2(b)). The total centroiding error was calculated as the Euclidean distance between the measured and correct star position. This function will be used to adjust the noise values in the Kalman Filter as was discussed in section III. The fitted function has the following form:

$$
\sigma_{t o t}=\frac{1}{a(1) * \sqrt{p o w}+a(2)}+a(3),
$$

where $\sigma_{\text {tot }}$ is the total centroiding error standard deviation, pow is the signal power, and the $a$-values are 


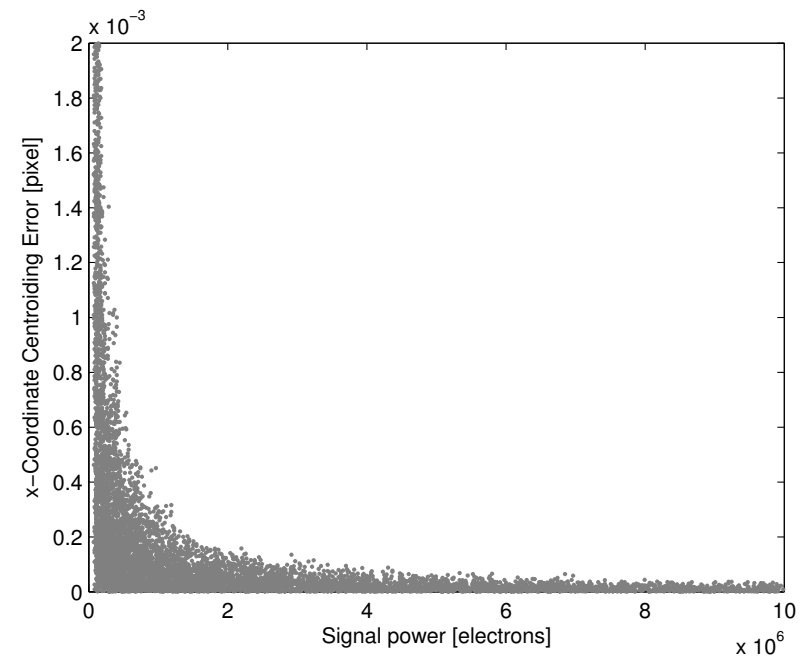

(a) Centroiding error

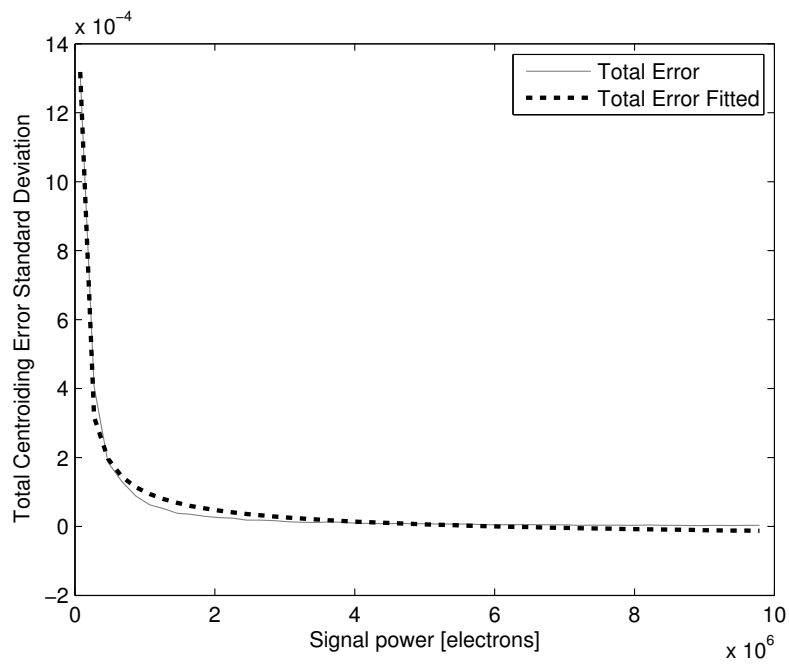

(b) Centroiding error standard deviation with fitted model

Figure 2: The effect of the signal power on the centroiding error.

determined in a least squares function fitting method to fit the model to the data.

\section{The Point Spread Function Width}

To simulate the relation between the point spread function width of a star and the centroiding error, simulations were performed with a wide range of stars with different PSF widths. The centroiding algorithm was executed for these images and the centroiding error was set out in function of the PSF widths. The results are given that link the $x$-centroiding error with the PSF width in the $x$-direction and that link the $y$-centroiding error with the PSF width in the $y$-direction.

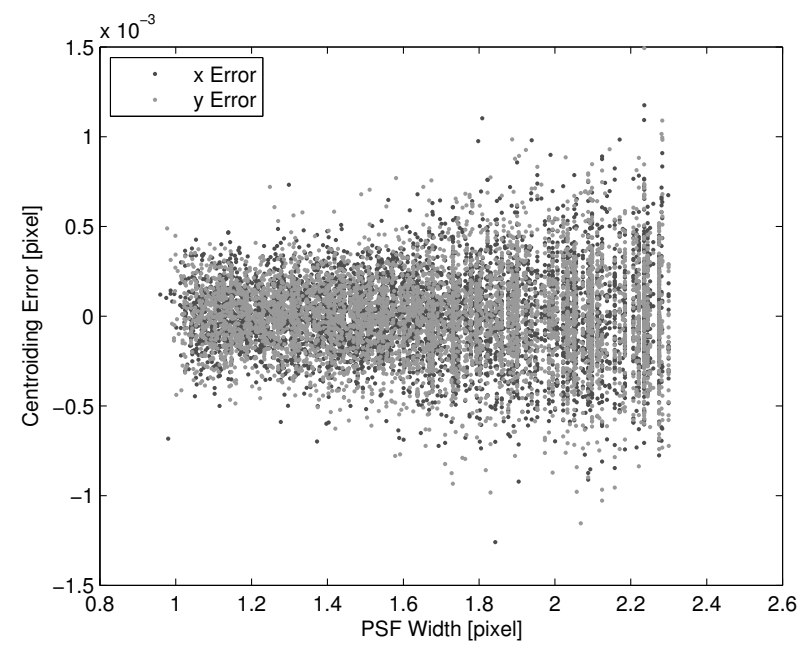

(a) Centroiding error

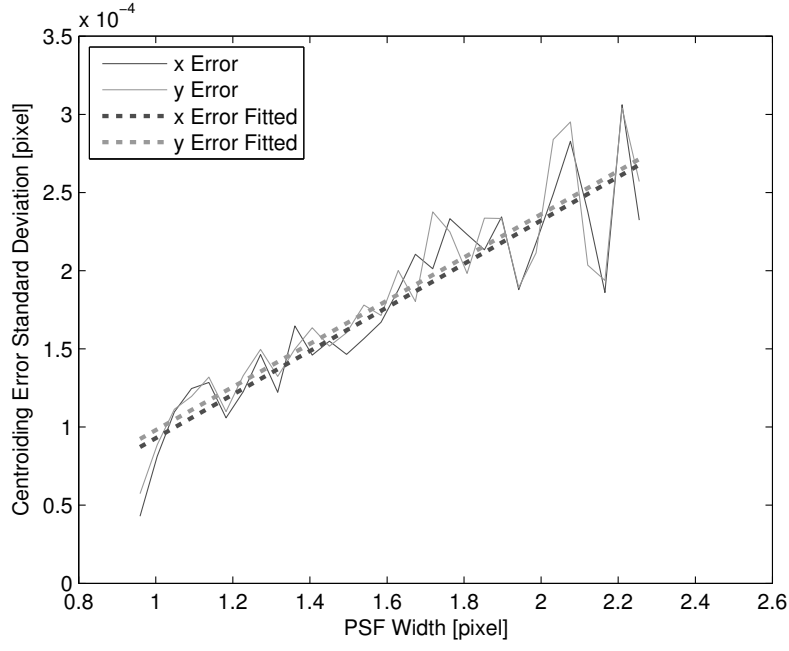

(b) Centroiding error standard deviation with fitted model

Figure 3: The effect of the PSF width on the centroiding error.

As can be seen from figure (a) of figure 3, the centroiding error increases with increasing width of the PSF. From figure (b), where the centroiding error standard deviation is set out in function of both the PSF width in $x$ and $y$ direction, it is clear that the relation is linear. A linear model can be fitted through the 
data. The values of this fitted function can be used to estimate the centroiding error based on the PSF width.

\section{Kalman Filter simulation results}

The centroiding Kalman Filter was simulated in its basic version of section II and with weights adjusted as discussed in section III. To adjust the weights, information about the power in the star signal was used. This information is used to adjust the values for $\sigma_{s t x_{k}}$ and $\sigma_{s t y_{k}}$ used in matrix $\mathbf{R}_{\mathbf{k}}$.

First, the function values ( $a$-values) of equation 24 were determined by analysing the relationship between signal power and the true total centroiding error standard deviation. The $\sigma$-values were then calculated for each star during each time step by using equation 24 with the determined $a$-values and the measured power in the signal. Because the function values are unreliably at lower signal powers, the final values for $\sigma_{s t x_{k}}$ and $\sigma_{s t y_{k}}$ were calculated as a compromise between these calculated function values and the average values for $\sigma_{s t x_{k}}$ and $\sigma_{s t y_{k}}$ which are also used in the basic Kalman filter. This way, the information derived from the signal power acts to adjust the average noise values. For a star with lower than average power, the noise values will go up, for a star with higher power, the noise values will go down. The final values for $\sigma_{s t x_{k}}$ and $\sigma_{s t y_{k}}$ were this way computed as a weighted average between the average noise values (with weight 0.7) and the calculated noise values based on the signal power (with weight 0.3). In figure 4 , the root mean square of the centroiding errors of all stars is shown for each time step. These centroiding errors are shown for the raw centroiding measurements and for the Kalman Filter with and without noise adjustment.

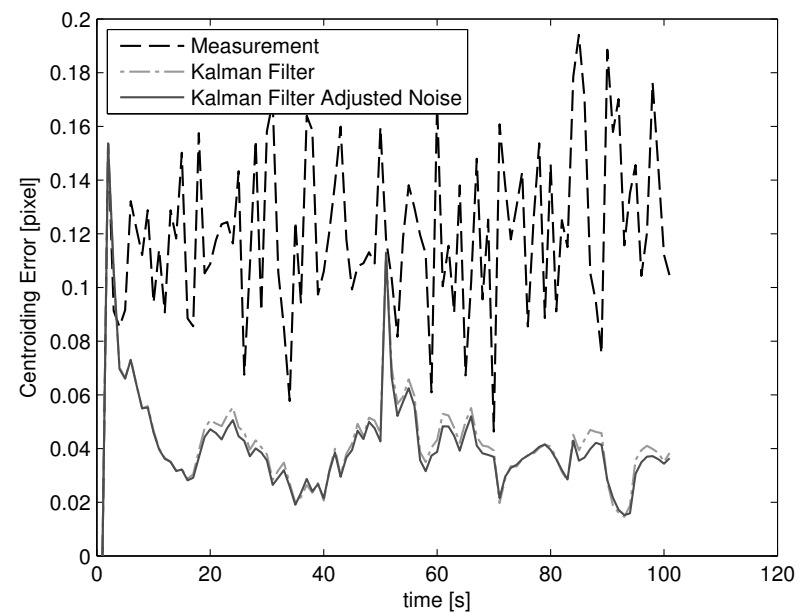

(a)

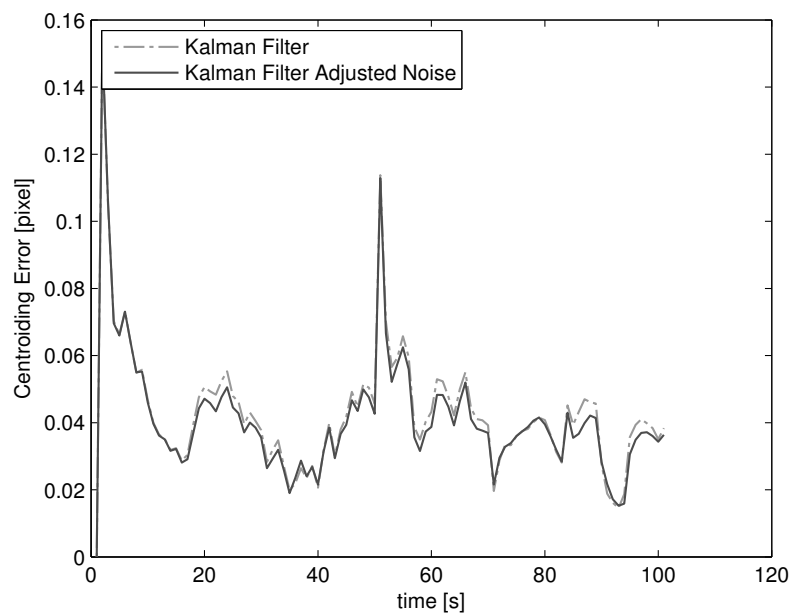

(b) Detail

Figure 4: The star position estimation error with and without adjustment of the noise.

From figure $4 \mathrm{~b}$, it follows that adjusting the noise values based on the signal power decreases the centroiding error. The error goes down slightly for almost every time step. This reduction in centroiding error leads to a more accurate attitude estimate.

\section{Uncertainties and Weights}

In this section, the impact of adapting the weights in the cost function of the attitude estimation algorithm is analysed. In these simulations, the AIM algorithm is used because it allows to easily incorporate different weights for the $x$ - and $y$-coordinate of the stars. The weights were simply calculated by taking the inverse of the square root of the diagonal elements of the covariance matrix $\mathbf{P}_{\mathbf{k}}$ : 


$$
\begin{aligned}
& w_{k_{x}}=\frac{1}{\sqrt{P_{x x_{k}}}} \\
& w_{k_{y}}=\frac{1}{\sqrt{P_{y y_{k}}}}
\end{aligned}
$$

We first verify whether or not these weights, based on the estimated covariance, give an accurate idea about the true star position estimation error. In figure 5, the true star position estimation error is shown for each of the stars. This value is the result of taking the root mean square of all estimation errors over the length of the simulation. In the dotted lines, the weights that are assigned to each star are given. These weights together sum to one.

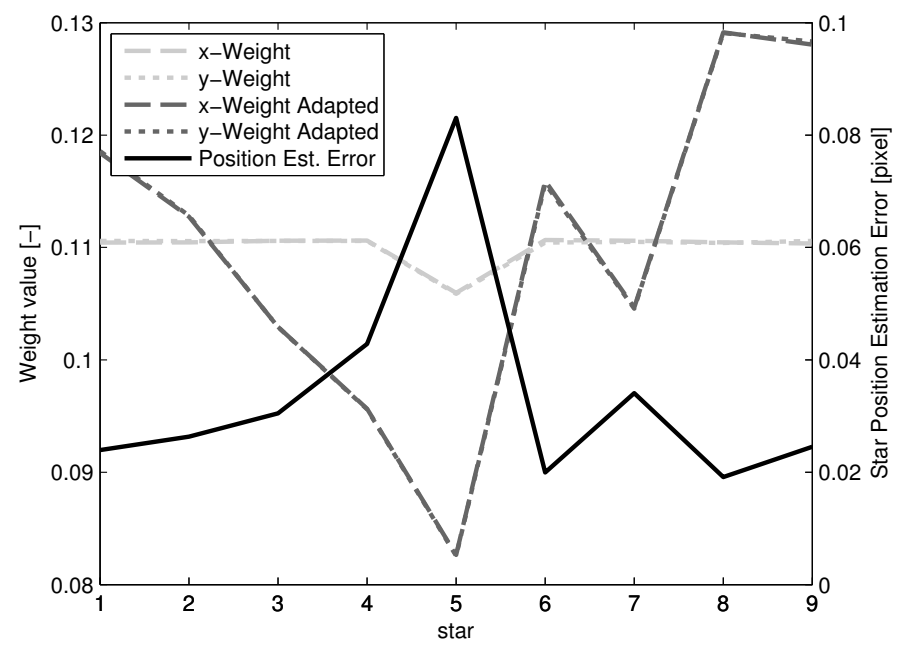

Figure 5: The weights and position estimation error for each star pair.

When the values in the matrices $\mathbf{Q}_{\mathbf{k}}$ and $\mathbf{R}_{\mathbf{k}}$ are chosen to be constant, as is the case in the basic Kalman Filter, the weights are almost the same for every star. This is because with the same $\mathbf{Q}_{\mathbf{k}}$ and $\mathbf{R}_{\mathbf{k}}$ matrices for each star, there is little difference in the covariance estimation cycle. This is the case for the light gray lines in figure 5. The main outlier is star number five. The reason for the lower weight assigned to this star is that the star disappeared from the image at time step 50 and another star needed to be tracked. This can be seen in figure 6 . Because the uncertainty is high for a new star, the covariance value is initially higher and the weight assigned to this star is lower. Over the total course of the simulation, this star therefore gets a lower weight.

When the weights are adapted, the matrices $\mathbf{Q}_{\mathbf{k}}$ and $\mathbf{R}_{\mathbf{k}}$ and therefore also the calculation of the covariance change. When stars get larger values for $\sigma_{s t x_{k}}$ and $\sigma_{s t y_{k}}$, the values in matrix $\mathbf{P}_{\mathbf{k}}$ rise and the weights for that star become lower. From figure 5, it can be seen that the weights (dark gray dotted lines) are lower for stars with higher position estimation error and vice versa. This way, stars with a higher position error are given less importance in the attitude estimation algorithm and the overall accuracy increases.

\section{E. Results}

In this section, the results for the Kalman Filters with different adaptations are shown. The performance of these filters is evaluated based on the root mean square of the attitude estimation error (equation 23) of the entire simulation sequence. The lower this error, the more accurate the attitude estimate. The results for the different cases that are described above are given in table 2.

When the Raw Measurements of the centroiding algorithm are used to estimate the attitude, the attitude estimation error is 11.64 arc seconds. When the Kalman Filter is used, these results are improved because 


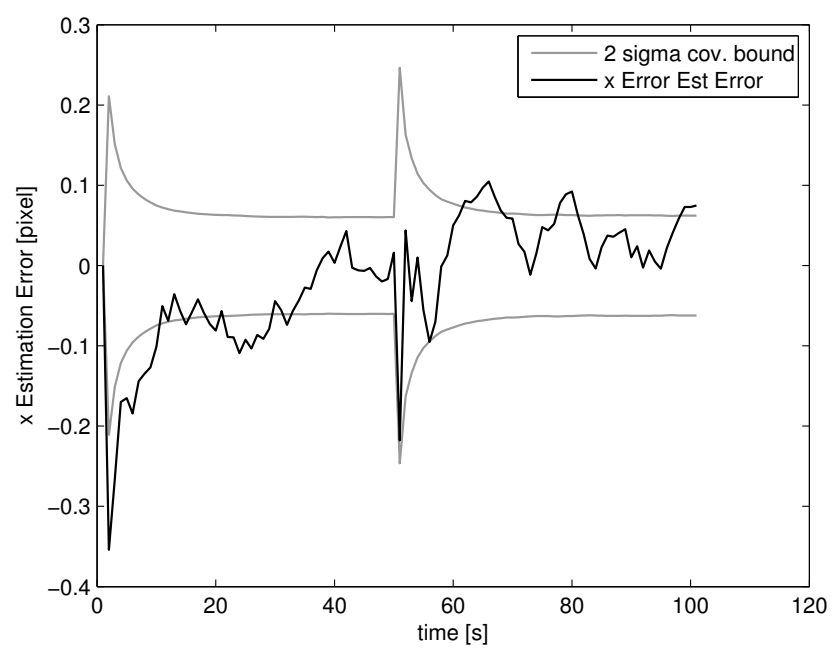

Figure 6: The x-coordinate estimation error and estimation covariance for a changing star.

Table 2: The values used to generate the data sets

\begin{tabular}{lc}
\hline \hline Case & Results (arcsec) \\
\hline Raw Measurement & 11.64 \\
Filter & 3.13 \\
Filter with Adjusted Noise & 3.10 \\
Filter with Adjusted Attitude Estimation Weights & 3.01 \\
Filter with Adjusted Noise and Attitude Estimation Weights & 2.71 \\
\hline \hline
\end{tabular}

the gyroscope information is added. With the basic Kalman Filter, the attitude estimation error is 3.13 arc seconds.

Adjusting the noise values based on the signal power information increases the accuracy slightly. The effect is quite small because adjusting the noise mainly helps to improve the accuracy of the Kalman gain. In this case, the filter mainly assigns importance to the gyroscope measurements (the diagonal elements in $\mathbf{K}_{\mathbf{k}}$ are around 0.1). Because of this, assigning slightly more or less importance to the star tracker measurements has a limited effect.

Using adjusted weights in the attitude estimation procedure (without adjusting the noise values) reduces the attitude estimation error to 3.01 arc seconds. In the example simulation sequence of this example, this is because star number five is given less importance after its renewal. Because the star had high centroiding errors, the effect of these errors is diminished thanks to the lower weights.

When both the noise and weights are adjusted, the error goes down to 2.71 arc seconds. Adjusting the noise not only helps to calculate a better Kalman gain, it also helps to calculate more accurate covariance matrices. This in turn improves the weights that can be used in the attitude estimation algorithm. Because of this effect, the attitude estimation error is decreased significantly.

\section{Conclusion}

In this paper, methods to improve the accuracy of star position estimation using a Kalman Filter were discussed. A Kalman Filter with low computational complexity is presented to fuse the information of the on-board gyroscopes with that of the star tracker centroiding algorithm. It was shown that in the 
centroiding procedure, sources of information are available that allow to improve our knowledge of the expected centroiding error. Using values such as the total signal power, and the PSF width, the expected centroiding errors for different stars can be determined. This way, the Kalman Filter can assign more importance to the gyroscope measurements for stars with higher expected centroiding errors, and vice versa. In simulations, it was shown that adjusting the Kalman Filter values to incorporate this information, can increase the accuracy of the star position estimation. Furthermore, the Kalman Filter provides a convenient method to estimate the uncertainty on each star position estimate. This can be used to determine weights for each star in the attitude estimation algorithms. This way, stars with higher uncertainty on their position estimate can be given a lower weight in the attitude estimation algorithm. The Kalman filter with the above mentioned adjustments was implemented and tested with simulated data. Results show that the attitude estimation accuracy can be significantly increased using these methods. This increased accuracy leads to a more accurate attitude determination and control system, which can allow to have more demanding spacecraft missions.

\section{Acknowledgement}

Tjorven Delabie is funded by the Agency for Innovation by Science and Technology in Flanders (IWT).

\section{References}

${ }^{1}$ Wertz, J. R., Spacecraft Attitude Determination and Control, D. Reidel Publishing Company, Dordrecht, Holland, 1978, ISBN 90-277-0959-9.

${ }^{2}$ Vandersteen, J., Observation and Estimation for Space Applications, Ph.D. thesis, KU Leuven, 2012.

${ }^{3}$ Liebe, C. C., "Accuracy Performance of Star Trackers - A Tutorial," IEEE Transactions on aerospace and electronic systems, Vol. 38, No. 2, 2002.

${ }^{4}$ Stone, R. C., "A Comparison of Digital Centering Algorithms," The Astronomical Journal, Vol. 97, No. 4, 1989.

${ }^{5}$ Vyas, A., Roopashree, M., and Prasad, B. R., "Improved Iteratively Weighted Centroiding for accurate spot detection in Laser Guide Star based Shack Harmann Sensor," Proceedings of SPIE, Vol. 7588, 2010.

${ }^{6}$ Auer, L. and Altena, W. V., "Digital Image Centering II," The Astronomical Journal, Vol. 83, No. 5, 1978.

${ }^{7}$ bo Liu, H., kun Yang, J., qi Wang, J., chun Tan, J., and jian Li, X., "Star spot location estimation using Kalman filter for star tracker," Vol. 50, 2011, pp. 1735-1744.

${ }^{8}$ Markley, F. L. and Mortari, D., "How to estimate attitude from vector observations," AAS/AIAA Astrodynamics Specialist Conf., No. 427 in 99, Aug. 1999.

${ }^{9}$ Delabie, T., "A Highly Efficient Attitude Estimation Algorithm for Star Trackers Based on Optimal Image Matching," AIAA Guidance, Navigation and Control Conference, Minneapolis, Minnesota, 2012.

${ }^{10}$ Feuchtgruber, H., "Herschel STR-A CCD Sub-Pixel Structure", Max Planck Institute for Extraterrestrial Physics PICCME-TN-041, Garching, Germany, March 2012.

${ }^{11}$ A, C. F., B, G. W. H., and B, B. E., "DETERMinATION OF CENTROID OF CCD STAR IMAGES," .

${ }^{12}$ Winick, K. A., "Cramér-Rao lower bounds on the performance of charge-coupled-device optical position estimators," Optical Society of America, Vol. 3, No. 11, 1986.

${ }^{13}$ Delabie, T., Vandenbussche, B., and Schutter, J. D., "An Accurate and Efficient Gaussian Fit Centroiding Algorithm for Star Trackers," AAS/AIAA Space Flight Mechanics Meeting, 2013.

${ }^{14}$ Sidi, M. J., Spacecraft Dynamics and Control: A Practical Engineering Approach, Cambridge University Press, 1997, ISBN: 0-512-55072-6.

${ }^{15}$ Farrenkopf, R., "Analytic steady-state accuracy solutions for two common spacecraft attitude estimators," Journal of Guidance, Navigation and Control,, Vol. 1, 1944, pp. 282-284.

${ }^{16}$ IEEE, "IEEE Standard for Inertial Sensor Terminology," 2001.

${ }^{17}$ ESA, "The Hipparcos and Tycho Catalogues," ESA SP-1200, 1997. 